\title{
Gastrointestinal Hemorrhage due to Metastatic Choriocarcinoma
}

\author{
Ayesha J Khan ${ }^{1}$, Gopal S Bhargawa ${ }^{2}$, Manu Kohli ${ }^{3}$, Kulwant S Ded ${ }^{4}$
}

\begin{abstract}
Testicular germ-cell tumors represent approximately $1 \%$ of all cancers in man and are the most common malignancy between 15- and 35-year olds. Choriocarcinoma represents less then $1 \%$ of testicular tumors. Metastatic choriocarcinoma is a rare nonseminomatous germ-cell tumor with a characteristic hemorrhagic tendency due to its trophoblastic origin. The main characteristic of these tumors is that they metastasize to retroperitoneal lymph nodes, less frequently, to lungs, liver, and brain in $80 \%$ of the cases. Metastasis to gastrointestinal tract is extremely rare, around $5 \%$. We report a case of 39-year-old man with no significant medical or surgical history was admitted with complaints of melaena and was diagnosed by the presence of metastatic lesions in the jejunum and ileum during laparotomy and left high-end orchidectomy was done. The patient could not survive because of grave prognosis. This is probably due to choriocarcinoma on histological findings. In conclusion, the case of gastrointestinal hemorrhage, a pan endoscopic examination, and histologic evaluation should be carried out to identify metastatic lesions and provide potential endoscopic therapy.
\end{abstract}

Keywords: Gastrointestinal hemorrhage, Germ-cell tumors, Metastatic choriocarcinoma.

AMEI's Current Trends in Diagnosis \& Treatment (2019): 10.5005/jp-journals-10055-0061

\section{INTRODUCTION}

Tumoral metastatic disease involving the gut is an infrequent event that may result in gastrointestinal hemorrhage. In most cases, a diagnosis is casually reached during autopsy, radiological techniques, or surgical procedures. Tumors most frequently associated with gut metastases include melanoma, lung and breast cancer, renal cell carcinoma, germ-cell tumors, and head and neck tumors. ${ }^{1}$ In patients with wide-spread tumoral disease and gastrointestinal hemorrhage, endoscopic management should be individualized according to personal and clinical characteristics, in order to obtain etiological information on the hemorrhage, a histological examination of potential bleeding lesions, and endoscopic therapy when necessary.

\section{Case Description}

A 39-year-old man with no significant medical or surgical history was admitted with complaints of melaena, dull aching pain in abdomen, shortness of breath, headache, fatigue, weight loss, fever up to $38^{\circ} \mathrm{C}$, since the last one month. History was unremarkable including no prior history of personal or family history of cancers or thrombotic conditions, recent surgery, or trauma. He was chronic alcoholic. Physical examination revealed a pale patient with bilateral lower extremity oedema without tenderness or erythema. On abdominal examination, diffuse dull aching pain was present. Testicular examination showed a firm left scrotal swelling, twice the size of right testis, with nosensation. Laboratory testing showed significant anaemia with hemoglobin of $5.7 \mathrm{~g} / \mathrm{dL}$, white blood count of $7,200 / \mu \mathrm{L}$, platelet count 2.6 lakhs/ $\mu \mathrm{L}$. Electrolytes and hepatic function were within normal limits.

On chest X-ray, mulitiple rounded opacities were seen in both lungs, and an abdominal computed tomography scan demonstrated a few lymph nodes in the retrocaval and in the left paraaortic region, measuring up to $24 \times 20 \times 15 \mathrm{~mm}$, mild hepatosplenomegaly with slightly hypodense lesions were seen in lumbar spines. On admission, the patient was presented with melaena and

\footnotetext{
${ }^{1-4}$ Department of Surgery, Sri Guru Ram Das Institute of Medical Sciences and Research, Amritsar, Punjab, India

Corresponding Author: Ayesha J Khan, Department of Surgery, Sri Guru Ram Das Institute of Medical Sciences and Research, Amritsar, Punjab, India, Phone: +919953251100, e-mail: drayeshakhan2025@ gmail.com

How to cite this article: Khan AJ, Bhargawa GS, Kohli M, et al. Gastrointestinal Hemorrhage due to Metastatic Choriocarcinoma. AMEl's Curr Trends Diagn Treat 2019;3(1):37-38.

Source of support: Nil

Conflict of interest: None
}

hypotension, which required massive blood transfusion. Beta human chorionic gonadotropin was $>5000 \mathrm{mlU} / \mathrm{mL}$. Alpha-feto protein (AFP) was $2.26 \mathrm{IU} / \mathrm{mL}(0.5-5.5 \mathrm{IU} / \mathrm{mL})$ with no positivity for other tumor markers. Upper gastrointestinal (GI) endoscopy revealed grade A esophagitis, with no evidence of blood. Due to the absence of blood in the upper endoscopy, a colonoscopy was performed which was normal. Magnetic resonance imaging of the brain showed hemorrhagic metastasis. Ultrasonography (USG) scrotum showed a large well-defined heterogenous lesion of size $8.9 \times 5.5 \mathrm{~cm}$ with few anechoic areas within it seen in the left scrotal sac. Left testis and epididymis were not separately defined with the evidence of size $3.5 \times 3 \mathrm{~cm}$ smoothly marginated hypoechoic area with thin internal septation within it. The left spermatic cord appears thickened and echogenic. Stool for occult blood was positive. After getting multiple blood transfusions, patient was taken up for surgery-explorative laparotomy in which around 20 $\mathrm{cm}$ ileal resection was done (Fig. 1) and left high-end orchidectomy was done (Fig. 2). Histopathological findings were consistent with a choriocarcinoma. He was staged as stage 3A, T3N2M1a given metastasis to small intestine, lungs, and brain. Unfortunately for the patient, the fast installation of multiple metastatic injuries 


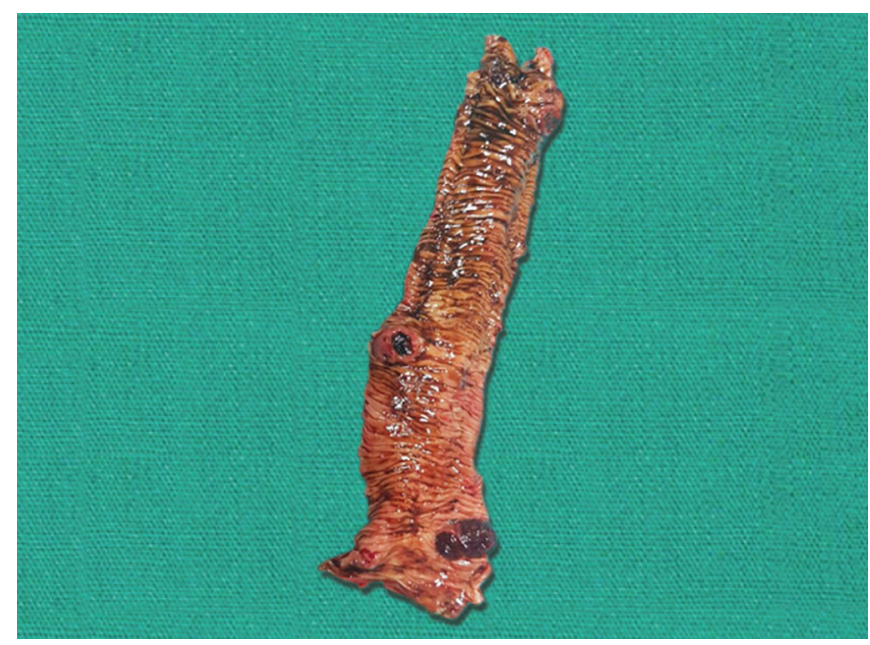

Fig. 1: Specimen of $20 \mathrm{~cm}$ ileum after exploratory lapratomy

led to his death before he could receive the proper treatment, which is combined chemotherapy with a high dose of cisplatinum, etoposide, and bleomicine, aside from the surgical treatment.

\section{Discussion}

Nongestational choriocarcinoma is a rare trophoblastic germ-cell tumor with a characteristically aggressive spread through the lymph and blood from the retroperitoneal space, mediastinum, and gonads. Because of its origin, metastatic lesions produce $\beta$-human chorionic gonadotropin; they also have a hemorrhagic tendency that results in the disease often presenting with bleeding from metastatic lesions. Metastatic disease is usually located in the lung, brain, bones, and lymph nodes, and it involves the gut in less than $5 \%$ of cases. ${ }^{2}$ In such cases, the stomach, usually the upper body, is the most common location, with a few case reports on the small intestine and colon. ${ }^{3-6}$ In any metastatic neoplasm, implantation takes place in the submucosa once tumor cells reach the gastrointestinal tract, as they fail to pass through the capillary barrier. As this submucosal tumor grows, blood supply to its central area decreases, which gives rise to erosions and ulcerations. For this reason, the main morphologic endoscopic features of metastatic gastrointestinal lesions are ulcerated submucosal and polypoid masses, ${ }^{1}$ which may require a more aggressive biopsy technique in order to obtain a proper histological material. Tumoral specific markers are an important diagnostic tool. $\beta$-Human chorionic gonadotropin serum concentrations are increased in all patients with pure choriocarcinoma, and the a-fetoprotein concentrations may be elevated in 30-60\% of choriocarcinomas, usually in mixed histological forms with other germ-cell tumors. Metastatic choriocarcinoma is a malignant disease with a high remission rate and a 5 -year overall survival rate of $70-80 \%$. Main prognostic factors include chemorefractory status, beta human gonadotropin serum concentrations higher than $1000 \mathrm{mlU} / \mathrm{mL}$, and a primary mediastinal tumor. However, the occurrence of gastrointestinal hemorrhage due to metastatic lesions and also promoted by chemotherapy adverse effects worsens the prognosis. The treatment of choice is a highdose cisplatin-based chemotherapy, and the role of coadjuvant peripheral blood stem-cell transplantation is being evaluated in clinical trials. ${ }^{7}$ The specific treatment of gastrointestinal bleeding metastatic disease is controversial because accurate topographic

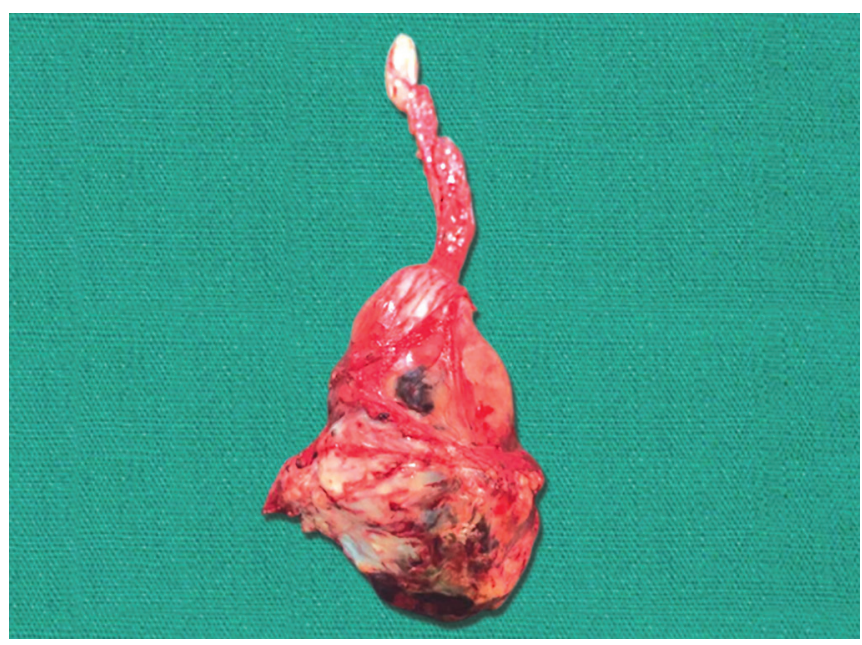

Fig. 2: Testis specimen after high-end orchidectomy

mapping of the lesions by endoscopy, laparoscopy, or angiography would be needed. In recent years, there have been some reports on laparoscopic surgery for isolated gastric and jejunal lesions $s^{3,8}$ and angiographic embolization, ${ }^{5}$ but no survival benefit has been proven with these approaches.

\section{Conclusion}

Testicular germ-cell tumor (GCT) is a malignancy of the young. In every young man with wide-spread, unidentified tumoral disease and various hemorrhagic symptoms, a germ-cell tumor should be ruled out by testicular echography, thoracic and abdominal scans, and $\beta$-human chorionic gonadotropin and $a$-fetoprotein serum concentrations. In the case of gastrointestinal hemorrhage, a panendoscopic examination and histologic evaluation should be carried out to identify metastatic lesions and provide potential endoscopic therapy; should this be not practical, angiography and surgery might prove useful for the control of active tumor bleeding.

\section{References}

1. Hsu C, Chen J, et al. Endoscopic features of metastatic tumors in the upper gastrointestinal tract. Endoscopy 1996;28:249-253. DOI: 10.1055/s-2007-1005437.

2. Sheinfeld J. Nonseminomatous germ cell tumors of the testis: current concepts and controversias. Urology 1994;44:2-14. DOI: 10.1016/ S0090-4295(94)80002-2.

3. Zerbib P, Prieur E, et al. Hemorrhagic digestive metastases from testicular choriocarcionoma. Ann Chir 2002;127:300-301. DOI: 10.1016/S0003-3944(02)00743-5.

4. Odelowo OO, Naab T, et al. Metastatic choriocarcinoma presenting as a bleeding duodenal ulcer. J Assoc Minor Phys 2001;12:144-148.

5. Rosenblatt GS, Walsh CJ, et al. Metastatic testis tumor presenting as gastrointestinal hemorrhage. J Urol 2000;164(5):1655. DOI: 10.1016/ S0022-5347(05)67057-7.

6. Garcia AO, Mahfoud C, et al. Acute low digestive hemorrhage in testicular choriocarcinoma. Acta Gastroenterol Latinoam 1997;27: 81-82.

7. Bokemeyer C, Harstrick A, et al. The use of dose- intensified chemotherapy in the treatment of metastatic nonseminomatous testicular germ cell tumors German Testicular Cancer Group. Semin Oncol 1998;25:24-32.

8. Galloway SW, Yeung EC, et al. Laparoscopic gastric resection for bleeding metastatic choriocarcinoma. Surg Endosc 2001;15(1):100. 